\title{
REMARKS ON THE UNIQUENESS PROBLEM FOR THE LOGISTIC EQUATION ON THE ENTIRE SPACE
}

\author{
YiHONG DU AND Lishan LiU
}

We consider the logistic equation $-\Delta u=a(x) u-b(x) u^{q}$ on all of $R^{N}$ with $a(x) /|x|^{\gamma}$ and $b(x) /|x|^{\tau}$ bounded away from 0 and infinity for all large $|x|$, where $\gamma>-2, \tau \in(-\infty, \infty)$. We show that this problem has a unique positive solution. This considerably improves some earlier results. The main new technique here is a Safonov type iteration argument. The result can also be proved by a technique introduced by Marcus and Veron, and the two different techniques are compared.

\section{INTRODUCTION}

We consider the logistic elliptic equation

$$
-\Delta u=a(x) u-b(x) u^{q}, \quad x \in R^{N},
$$

where $q$ is a constant greater than $1, a(x)$ and $b(x)$ are continuous functions with $b(x)$ positive on $R^{N}$. Equations of this kind have attracted extensive study because of interests in mathematical biology and Riemannian geometry. We refer to $[1,3,6,7,9,10,15]$ and the references therein for some of the previous research.

When the limits

$$
a_{\infty}=\lim _{|x| \rightarrow \infty} a(x) \text { and } b_{\infty}=\lim _{|x| \rightarrow \infty} b(x)
$$

exist and are positive numbers, it was shown in [9] that problem (1.1) has a unique positive solution $u$, and moreover,

$$
u(x) \rightarrow\left(a_{\infty} / b_{\infty}\right)^{1 /(q-1)} \text { as }|x| \rightarrow \infty .
$$

In [7], this result was extended to cases where these limits may not exist. Suppose that for some $\gamma \geqslant 0$, there exist positive numbers $\alpha_{1}, \alpha_{2}$ and $\beta_{1}, \beta_{2}$ such that

$$
\begin{array}{lll}
\alpha_{1}=\varliminf_{|x| \rightarrow \infty} \frac{a(x)}{|x|^{\gamma}}, & \alpha_{2}=\varlimsup_{|x| \rightarrow \infty} \frac{a(x)}{|x|^{\gamma},} \\
\beta_{1}=\varliminf_{|x| \rightarrow \infty} b(x), & \beta_{2}=\varlimsup_{|x| \rightarrow \infty} b(x) .
\end{array}
$$

\footnotetext{
Received 19th October, 2005
}

The first author was partially supported by the Australian Research Council. The second author was supported by the National Natural Science Foundation of China (10471075) and the Natural Science Foundation of Shandong Province of China (Y2003A01).

Copyright Clearance Centre, Inc. Serial-fee code: 0004-9727/06 \$A2.00+0.00. 
It is easily seen that under these conditions, (1.1) has at least one (weak) positive solution. By standard regularity theory of elliptic equations ([11]), any $W_{l o c}^{1,2}\left(R^{N}\right)$ solution of (1.1) belongs to $C^{1}\left(R^{N}\right)$.

It was proved in [7] that if $u \in C^{1}\left(R^{N}\right)$ is a positive solution of $(1.1)$, and if (1.2) is satisfied, then

$$
\varliminf_{|x| \rightarrow \infty} \frac{u(x)}{|x|^{\gamma /(q-1)}} \geqslant\left(\frac{\alpha_{1}}{\beta_{2}}\right)^{1 /(q-1)}, \varlimsup_{|x| \rightarrow \infty} \frac{u(x)}{|x|^{\gamma /(q-1)}} \leqslant\left(\frac{\alpha_{2}}{\beta_{1}}\right)^{1 /(q-1)} .
$$

If in addition, we suppose that

$$
q \frac{\alpha_{1} \beta_{1}}{\alpha_{2} \beta_{2}}>1
$$

then (1.1) has a unique positive solution. The techniques in [7] were partly motivated by $[3,15]$.

In this paper, we shall show that this uniqueness result holds without the extra condition (1.3). Moreover, we can relax condition (1.2) to the following:

There exist

$$
\gamma>-2, \tau \in(-\infty, \infty),
$$

and positive numbers $\alpha_{1}, \alpha_{2}$ and $\beta_{1}, \beta_{2}$ such that

$$
\begin{array}{ll}
\alpha_{1}=\varliminf_{|x| \rightarrow \infty} \frac{a(x)}{|x|^{\gamma}}, & \alpha_{2}=\varlimsup_{|x| \rightarrow \infty} \frac{a(x)}{|x|^{\gamma},} \\
\beta_{1}=\varliminf_{|x| \rightarrow \infty} \frac{b(x)}{|x|^{\tau}}, & \beta_{2}=\varlimsup_{|x| \rightarrow \infty} \frac{b(x)}{|x|^{\tau}} .
\end{array}
$$

Condition (1.4) was first used in [6]. It follows from [6, Corollary 3.5] that under (1.4), equation (1.1) has a minimal and a maximal positive solution. Here, our uniqueness result implies that these solutions coincide.

In section 2, we use an iteration argument motivated by one attributed to Safonov (in an unpublished article) to prove the uniqueness result. For boundary blow-up solutions over a bounded domain, various versions of this technique have been successfully used in uniqueness proofs in $[12,4,8,5]$. Here we show that this technique can also be used for entire space problems.

We would like to remark that, in proving uniqueness results for boundary trace problems over a bounded domain, Marcus and Veron ([13]) introduced a different technique, which can be used to prove, among other things, similar uniqueness results to those in $[12,4,8]$. However, it seems difficult to apply to problems where the nonlinearity is not necessarily convex, such as those treated in [5].

In section 3, we adapt the Marcus-Veron technique to give an alternative proof of our uniqueness result, which turns out to be much shorter than the one given in section 
2. Nevertheless, our proof in section 2 (which is the one we found first) can be extended to cases where the nonlinearity is not necessarily convex (see Remark 2.4), besides other possible applications. So it seems worthwhile to publish that proof.

The assumption that $\gamma>-2$ in (1.4) plays an important role in our proofs. If $a(x) \leqslant C|x|^{\gamma}$ with $\gamma<-2$ for some $C>0$ and all large $|x|$, and $a(x)$ is positive somewhere in $R^{N}$, and $b(x)>0$ in $R^{N}$, then the results in [10] apply and by Theorem 1 there, there exists a unique $\sigma_{1}>0$ such that

$$
-\Delta u=\lambda a(x) u-b(x) u^{q}, x \in R^{N}
$$

has a unique positive solution $u \in H$ when $\lambda>\sigma_{1}$, and there is no such solution when $0<\lambda \leqslant \sigma_{1}$, where $H$ denotes the completion of $C_{0}^{1}\left(R^{N}\right)$ under the norm

$$
\left(\int_{R^{N}}|\nabla u|^{2} d x\right)^{1 / 2}
$$

It is unclear whether there are positive solutions outside $H$, but for the special case $b(x) \equiv 1$ and $q=2$, it was shown in [1] that, indeed, there are no other positive solutions.

\section{Main Result and its PROOF}

We first recall a comparison principle (see, for example, [9, Lemma 2.1]) which will be used in the later proof.

LEMMA 2.1. (Comparison principle) Suppose that $\Omega$ is a bounded domain in $R^{N}$. Let $u_{1}, u_{2} \in C^{1}(\Omega)$ be positive in $\Omega$ and satisfy (in the weak sense)

$$
\Delta u_{1}+a(x) u_{1}-b(x) u_{1}{ }^{q} \leqslant 0 \leqslant \Delta u_{2}+a(x) u_{2}-b(x) u_{2}{ }^{q} \text { in } \Omega
$$

and

$$
\varliminf_{d(x, \overline{\partial \Omega}) \rightarrow 0}\left(u_{2}-u_{1}\right) \leqslant 0 .
$$

where $q>1, a(x), b(x)$ are continuous with $b(x)$ positive on $\Omega$ and $\|a\|_{L^{\infty}(\Omega)}<\infty$. Then $u_{2} \leqslant u_{1}$ in $\Omega$.

It should be noted that in Lemma 2.1, the assumption that $u_{1}$ and $u_{2}$ are positive and satisfy (2.1) in $\Omega$ has hidden restrictions on $a(x)$ and $b(x)$. Moreover, from the proof in [9] one easily sees that the restriction that $u_{1}, u_{2} \in C^{2}(\Omega)$ there can be replaced by $u_{1}, u_{2} \in C^{\mathbf{l}}(\Omega)$.

It follows from [6, Theorem 1] that, if (1.4) holds, then any positive solution $u \in C^{1}\left(R^{N}\right)$ of (1.1) satisfies

$$
\varliminf_{|x| \rightarrow \infty} \frac{u^{q-1}(x)}{|x|^{\gamma-\tau}} \geqslant \frac{\alpha_{1}}{\beta_{2}}, \quad \varlimsup_{|x| \rightarrow \infty} \frac{u^{q-1}(x)}{|x|^{\gamma-\tau}} \leqslant \frac{\alpha_{2}}{\beta_{1}} .
$$

The following technical lemma is the core of our iteration argument to be used in the uniqueness proof. 
LEMMA 2.2. Suppose that (1.4) holds and $u_{1}, u_{2}$ are positive solutions of (1.1). Then there exists $R>1$ large so that, if $x_{*} \in R^{N}$ satisfies, for some $k_{*} \geqslant k>1$,

$$
\left|x_{*}\right|>R, \quad u_{2}\left(x_{*}\right)>k_{*} u_{1}\left(x_{*}\right),
$$

then we can find $y_{*} \in R^{N}$, and positive constants $c_{0}=c_{0}(R, k)$ and $r_{0}=r_{0}(R, k)$ independent of $x_{*}$ and $k_{*}$, such that

$$
\left|y_{*}-x_{*}\right|=r_{0}\left|x_{*}\right|^{-\gamma / 2}, u_{2}\left(y_{*}\right)>\left(1+c_{0}\right) k_{*} u_{1}\left(y_{*}\right) .
$$

Proof: By (1.4) and (2.2), for all large $R>1$ and $|x|>R$,

$$
(1 / 2) \alpha_{1}|x|^{\gamma}<a(x)<2 \alpha_{2}|x|^{\gamma}, \quad(1 / 2) \beta_{1}|x|^{\top}<b(x)<2 \beta_{2}|x|^{\top},
$$

and, for $i=1,2$,

$$
\mu_{1}|x|^{(\gamma-\tau) /(q-1)}<u_{i}(x)<\mu_{2}|x|^{(\gamma-\tau) /(q-1)}
$$

where

$$
\mu_{1}=(1 / 2)\left(\frac{\alpha_{1}}{\beta_{2}}\right)^{1 /(q-1)}, \mu_{2}=2\left(\frac{\alpha_{2}}{\beta_{1}}\right)^{1 /(q-1)} .
$$

We now fix $R>1$ large enough so that $R^{-1-(\gamma / 2)}<1 / 2$ and $(2.4),(2.5)$ hold for all $x$ satisfying $|x|>R / 2$. Then we define

$$
\Omega_{0}:=\left\{x \in R^{N}: u_{2}(x)>k_{*} u_{1}(x)\right\} \cap B_{\tau}\left(x_{*}\right),
$$

where

$$
r=r_{0}\left|x_{*}\right|^{-\gamma / 2}, B_{r}\left(x_{*}\right)=\left\{x \in R^{N}:\left|x-x_{*}\right|<r\right\},
$$

and $r_{0} \in(0,1)$ is to be determined below.

Clearly $x \in \Omega_{0}$ implies

$$
\left|x_{*}\right|-r \leqslant|x| \leqslant\left|x_{*}\right|+r
$$

which in turn implies, due to $\left|x_{*}\right|>R$ and our choice of $R$,

$$
(1 / 2)\left|x_{*}\right|<|x|<(3 / 2)\left|x_{*}\right|
$$

We now consider $u_{2}-k_{*} u_{1}$ in $\Omega_{0}$. Using (2.4), (2.5) and (2.6) and the assumption that $u_{2}>k * u_{1}$ in $\Omega_{0}$, we deduce, for $x \in \Omega_{0}$,

$$
\begin{aligned}
\Delta\left(u_{2}-k_{*} u_{1}\right) & =-a(x)\left(u_{2}-k_{*} u_{1}\right)+b(x)\left(u_{2}^{q}-k_{*} u_{1}^{q}\right) \\
& \geqslant-a(x)\left(u_{2}-k_{*} u_{1}\right)+b(x)\left(k_{*}^{q} u_{1}^{q}-k_{*} u_{1}^{q}\right) \\
& \geqslant-2 \alpha_{2}|x|^{\gamma}\left(u_{2}-k_{*} u_{1}\right)+(1 / 2) \beta_{1}|x|^{\gamma} u_{1}^{q}\left(k_{*}^{q}-k_{*}\right) \\
& \geqslant-2 \alpha_{2}|x|^{\gamma}\left(u_{2}-k_{*} u_{1}\right)+(1 / 2) \beta_{1} \mu_{1}^{q}|x|^{\tau+q(\gamma-\tau) /(q-1)}\left(k_{*}^{q}-k_{*}\right) \\
& \geqslant-M\left|x_{*}\right|^{\gamma}\left(u_{2}-k_{*} u_{1}\right)+m k_{*}\left|x_{*}\right|^{\sigma}
\end{aligned}
$$


where

$$
\begin{aligned}
M & =2 \alpha_{2} \max \left\{(1 / 2)^{\gamma},(3 / 2)^{\gamma}\right\}, \sigma=\tau+q(\gamma-\tau) /(q-1) \\
m & =(1 / 2) \beta_{1} \mu_{1}^{q}\left(k^{q-1}-1\right) \min \left\{(1 / 2)^{\sigma},(3 / 2)^{\sigma}\right\}
\end{aligned}
$$

With these preparations, we now define

$$
w(x)=(2 N)^{-1} m k_{*}\left|x_{*}\right|^{\sigma}\left(r^{2}-\left|x-x_{*}\right|^{2}\right) .
$$

Clearly $w(x)>0$ in $B_{r}\left(x_{*}\right)$ and $\Delta w=-m k_{*}\left|x_{*}\right|^{\sigma}$. It follows that, for $x \in \Omega_{0}$,

$$
\Delta\left(u_{2}-k_{*} u_{1}+w\right) \geqslant-M\left|x_{*}\right|^{\gamma}\left(u_{2}-k_{*} u_{1}\right) \geqslant-M\left|x_{*}\right|^{\gamma}\left(u_{2}-k_{*} u_{1}+w\right) .
$$

If we denote by $\lambda_{1}(\Omega)$ the first eigenvalue of $-\Delta$ over $\Omega$ under homogeneous Dirichlet boundary conditions, we have

$$
\lambda_{1}\left(\Omega_{0}\right) \geqslant \lambda_{1}\left(B_{r}\left(x_{*}\right)\right)=r^{-2} \lambda_{1}\left(B_{1}\left(x_{*}\right)\right)
$$

Therefore

$$
\lambda_{1}\left(\Omega_{0}\right) \geqslant r_{0}^{-2}\left|x_{*}\right|^{\gamma} \lambda_{1},
$$

where $\lambda_{1}=\lambda_{1}\left(B_{1}\left(x_{*}\right)\right)$ is independent of $x_{*}$. We now choose $r_{0} \in(0,1)$ small enough so that

$$
r_{0}^{-2} \lambda_{1}>M \text { and hence } \lambda_{1}\left(\Omega_{0}\right)>M\left|x_{*}\right|^{\gamma} \text {. }
$$

Then by the maximum principle (see [2]), due to (2.7),

$$
u_{2}\left(x_{*}\right)-k_{*} u_{1}\left(x_{*}\right)+w\left(x_{*}\right) \leqslant \max _{\partial \Omega_{0}}\left(u_{2}-k_{*} u_{1}+w\right) \text {. }
$$

We observe that the maximum of $\left(u_{2}-k_{*} u_{1}+w\right)$ over $\partial \Omega_{0}$ has to be achieved by some $y_{*} \in \partial B_{r}\left(x_{*}\right)$ since any $y \in \partial \Omega_{0} \backslash \partial B_{r}\left(x_{*}\right)$ satisfies, by the definition of $\Omega_{0}, u_{2}(y)=k_{*} u_{1}(y)$ and hence

$$
u_{2}(y)-k_{*} u_{1}(y)+w(y)=w(y) \leqslant w\left(x_{*}\right)<u_{2}\left(x_{*}\right)-k_{*} u_{1}\left(x_{*}\right)+w\left(x_{*}\right)
$$

Thus we can find $y_{*} \in \partial \Omega_{0}$ satisfying $\left|y_{*}-x_{*}\right|=r$ (hence $w\left(y_{*}\right)=0$ ) such that

$$
\begin{aligned}
u_{2}\left(y_{*}\right)-k_{*} u_{1}\left(y_{*}\right) & =u_{2}\left(y_{*}\right)-k_{*} u_{1}\left(y_{*}\right)+w\left(y_{*}\right) \\
& \geqslant u_{2}\left(x_{*}\right)-k_{*} u_{1}\left(x_{*}\right)+w\left(x_{*}\right) \\
& >w\left(x_{*}\right)=(2 N)^{-1} m k_{*}\left|x_{*}\right|^{\sigma} r^{2} \\
& =(2 N)^{-1} m k_{*} r_{0}^{2}\left|x_{*}\right|^{(\gamma-\tau) /(q-1)} \\
& \geqslant c_{1} k_{*}\left|y_{*}\right|^{(\gamma-\tau) /(q-1)}
\end{aligned}
$$

where

$$
c_{1}=(2 N)^{-1} m r_{0}^{2} \min \left\{(1 / 2)^{-(\gamma-\tau) /(q-1)},(3 / 2)^{-(\gamma-\tau) /(q-1)}\right\}>0,
$$


and we have used (2.6). Making use of (2.5), we finally deduce

$$
u_{2}\left(y_{*}\right)-k_{*} u_{1}\left(y_{*}\right)>c_{1} k_{*}\left|y_{*}\right|^{(\gamma-\tau) /(q-1)} \geqslant c_{1} \mu_{2}^{-1} k_{*} u_{1}\left(y_{*}\right) .
$$

Therefore we can take $c_{0}=c_{1} \mu_{2}^{-1}$ and the proof if complete.

THEOREM 2.3. Suppose that (1.4) holds. Then (1.1) has a unique positive solution.

Proof: As mentioned before, by [6], under condition (1.4), equation (1.1) has at least one positive solution and any such solution satisfies (2.2). Suppose by way of contradiction that (1.1) has two different solutions $u_{1}$ and $u_{2}$. Let

$$
k_{1}=\varliminf_{|x| \rightarrow \infty} \frac{u_{1}(x)}{u_{2}(x)}, k_{2}=\varlimsup_{|x| \rightarrow \infty} \frac{u_{2}(x)}{u_{1}(x)} .
$$

By (2.2) we know that both $k_{1}$ and $k_{2}$ are finite. If $k_{1} \leqslant 1$ and $k_{2} \leqslant 1$, then for any $\varepsilon>0$ there exists $R_{\varepsilon}>0$ such that for all $x$ satisfying $|x|>R_{\varepsilon}$,

$$
u_{1}(x) \leqslant(1+\varepsilon) u_{2}(x), u_{2}(x) \leqslant(1+\varepsilon) u_{1}(x) .
$$

Since $(1+\varepsilon) u_{1}$ and $(1+\varepsilon) u_{2}$ are upper solutions of (1.1), we apply Lemma 2.1 over $\Omega=B_{R}(0), R>R_{\varepsilon}$, and deduce

$$
u_{1}(x) \leqslant(1+\varepsilon) u_{2}(x), u_{2}(x) \leqslant(1+\varepsilon) u_{1}(x), \forall x \in R^{N} .
$$

Letting $\varepsilon \rightarrow 0$ we obtain $u_{1} \equiv u_{2}$, contradicting our assumption that they are different solutions.

So necessarily $\max \left\{k_{1}, k_{2}\right\}>1$. Without loss of generality we may assume that $k_{2}>1$. Therefore there exist a constant $k \in\left(1, k_{2}\right)$ and a sequence $\left\{x_{n}\right\}$ such that

$$
\left|x_{n}\right| \rightarrow \infty, \quad u_{2}\left(x_{n}\right) / u_{1}\left(x_{n}\right)>k, n=1,2, \ldots .
$$

We are now in a position to apply Lemma 2.2. Let $R, r_{0}$ and $c_{0}$ be determined by Lemma 2.2. We recall that $R$ satisfies $R^{-1-(\gamma / 2)}<1 / 2$. We first find an integer $j>1$ such that

$$
\left(1+c_{0}\right)^{j} k>\sup _{|x|>R} \frac{u_{2}(x)}{u_{1}(x)}
$$

Since $\left|x_{n}\right| \rightarrow \infty$, we can then find $n_{0}$ large enough such that

$$
\left|x_{n_{0}}\right|(1 / 2)^{j}>R \text {. }
$$

Taking $x_{*}=x_{n_{0}}$ and $k_{*}=k$ in Lemma 2.2, we can find $y_{*}=y_{1}$ such that

$$
\left|y_{1}-x_{*}\right|=r_{0}\left|x_{*}\right|^{-\gamma / 2}, u_{2}\left(y_{1}\right)>\left(1+c_{0}\right) k u_{1}\left(y_{1}\right) \text {. }
$$


Clearly

$$
\left|y_{1}\right| \geqslant\left|x_{*}\right|-r_{0}\left|x_{*}\right|^{-\gamma / 2} \geqslant\left|x_{n_{0}}\right|\left(1-R^{-1-(\gamma / 2)}\right)>\left|x_{n_{0}}\right|(1 / 2)>R .
$$

We now take $x_{*}=y_{1}$ and $k_{*}=\left(1+c_{0}\right) k$ in Lemma 2.2, and we can find $y_{2}$ such that

$$
\left|y_{2}-y_{1}\right|=r_{0}\left|y_{1}\right|^{-\gamma / 2}, u_{2}\left(y_{2}\right)>\left(1+c_{0}\right)^{2} k u_{1}\left(y_{2}\right) \text {. }
$$

Let us note that

$$
\left|y_{2}\right| \geqslant\left|y_{1}\right|(1 / 2) \geqslant\left|x_{n_{0}}\right|(1 / 2)^{2}>R .
$$

We can repeat the above process until we obtain $y_{j}$, which satisfies

$$
u_{2}\left(y_{j}\right)>\left(1+c_{0}\right)^{j} k u_{1}\left(y_{j}\right),\left|y_{j}\right| \geqslant\left|x_{n_{0}}\right|(1 / 2)^{j}>R .
$$

Therefore

$$
\frac{u_{2}\left(y_{j}\right)}{u_{1}\left(y_{j}\right)} \geqslant\left(1+c_{0}\right)^{j} k>\sup _{|x|>R} \frac{u_{2}(x)}{u_{1}(x)} .
$$

This contradiction completes our proof.

REMARK 2.4. The arguments in this section can be extended to cases where the right hand side of (1.1) is more general. For example, suppose that, for $u>0, f(u) / u$ is increasing and $c_{1} \leqslant f(u) / u^{q} \leqslant c_{2}$ for some positive constants $c_{1}$ and $c_{2}$, and suppose furthermore that $\lim _{u \rightarrow 0} f(u) / u^{q}$ exists when $\gamma>\tau, \lim _{u \rightarrow \infty} f(u) / u^{q}$ exists when $\gamma<\tau$, and $c_{1}=c_{2}$ when $\gamma=\tau$. Then Theorem 2.3 remains true if $u^{q}$ is replaced by $f(u)$ in (1.1).

\section{An alternative proof by the Marcus-Veron technique}

In this section, we provide an alternative proof of Theorem 2.3 by making use of a technique introduced by Marcus and Veron in [13, page 226] (see also [14]), which relies on the convexity of the nonlinearity and hence does not seem easily extendable to cases as discussed in Remark 2.4. However, this proof is considerably simpler.

Suppose that (1.4) holds. By Lemma 3.1 and Proposition 3.4 of [6], we know that for all large $R$, the problem

$$
-\Delta v=a(x) v-b(x) v^{q},\left.v\right|_{\partial B_{R}(0)}=0
$$

has a unique positive solution $v_{R}$, and as $R$ increases to infinity, $v_{R}$ increases to a positive solution $u_{*}$ of (1.1). By Lemma 2.1, it is easily seen that $u_{*}$ is the minimal positive solution of (1.1), namely, any positive solution of (1.1) satisfies $u \geqslant u_{*}$.

Arguing indirectly, we assume that (1.1) has a positive solution $u$ such that $u \not \equiv u_{*}$. By the strong maximum principle, we easily deduce that $u>u_{*}$ in $R^{N}$. Due to (2.2), we can find a constant $k>1$ such that $u \leqslant k u$ in $R^{N}$.

We are now ready to apply the Marcus-Veron technique. Define

$$
v=u_{*}-(2 k)^{-1}\left(u-u_{*}\right) \text {. }
$$




\section{Clearly}

$$
u_{*}>v \geqslant \frac{k+1}{2 k} u_{*}, \quad \frac{2 k}{2 k+1} v+\frac{1}{2 k+1} u=u_{*} .
$$

Denote $f(x, t)=-a(x) t+b(x) t^{q}$. We find

$$
\frac{\partial^{2}}{\partial t^{2}} f(x, t) \geqslant 0
$$

for all $x \in R^{N}$ and $t>0$. Therefore $f(x, t)$ is convex in $t$ for $t>0$, and by (3.2),

$$
f\left(x, u_{*}\right) \leqslant \frac{2 k}{2 k+1} f(x, v)+\frac{1}{2 k+1} f(x, u) .
$$

It follows that

$$
-\Delta v=-\left(1+\frac{1}{2 k}\right) f\left(x, u_{*}\right)+\frac{1}{2 k} f(x, u) \geqslant-f(x, v),
$$

that is,

$$
-\Delta v \geqslant a(x) v-b(x) v^{q}
$$

We now apply Lemma 2.1 with $\Omega=B_{R}(0), u_{1}=v$ and $u_{2}=v_{R}$, where $v_{R}$ is the unique positive solution of (3.1). It follows that $v_{R} \leqslant v$ in $B_{R}$ for all large $R$, from which we deduce $u_{*} \leqslant v$ in $R^{N}$. But this is a contradiction to (3.2). This proves the uniqueness result.

\section{REFERENCES}

[1] G.A. Afrouzi and K.J. Brown, 'On a diffusive logistic equation', J. Math. Anal. Appl. 225 (1998), 326-339.

[2] H. Beresticky, L. Nirenberg and S.R.S. Varadhan, 'The principal eigenvalue and maximum principle for second order elliptic operators in general domains', Comm. Pure Appl. Math., 47 (1994), 47-92.

[3] B. Bianchini and M. Rigoli, 'Nonexistence and uniqueness of positive solutions of Yamabe type equations on nonpositively curved manifolds', Trans. Amer. Math. Soc. 349 (1997), 4753-4774.

[4] M. Chuaqui, C. Cortazar, M. Elgueta and J. Garcia-Melian, 'Uniqueness and boundary behaviour of large solutions to elliptic problems with singular weights', Commun. Pure Appl. Anal. 3 (2004), 653-662.

[5] F. Cirstea and Y. Du, 'General uniqueness results and variation speed for blow-up solutions of elliptic equations', Proc. London Math. Soc. 91 (2005), 459-482.

[6] W. Dong, 'Positive solutions for logistic type quasilinear elliptic equations on $R^{N}, J$. Math. Anal. Appl. 290 (2004), 469-480.

[7] W. Dong and Y. Du, 'Unbounded principal eigenfunctions and the logistic equation on $R^{N}$, Bull. Austral. Math. Soc. 67 (2003), 413-427.

[8] Y. Du, 'Asymptotic behaviour and uniqueness results for boundary blow-up solutions', Differential Integral Equations 17 (2004), 819-834. 
[9] Y. Du and L. Ma, 'Logistic type equations on $R^{N}$ by a squeezing method involving boundary blow-up solutions', J. London. Math. Soc. (2) 64 (2001), 107-124.

[10] Y. Du and L. Ma, 'Positive solutions of an elliptic partial differential equation on $R^{N}$, J. Math. Anal. Appl. 271 (2002), 409-425.

[11] D. Gilbarg and N.S. Trudinger, Elliptic partial differential equations of second order, (Second Edition) (Springer-Verlag, Berlin, 1983).

[12] S. Kim, 'A note on boundary blow-up problem of $\Delta u=u^{p '}$, (IMA preprint series No. 1872, Univ. of Minnesota, 2002).

[13] M. Marcus and L. Veron, 'The boundary trace of positive solutions of semilinear elliptic equations: the subcritical case', Arch. Rational Mech. Anal. 144 (1998), 201-231.

[14] M. Marcus and L. Veron, 'Existence and uniqueness results for large solutions of general nonlinear elliptic equations', J. Evol. Eqns. 3 (2003), 637-652.

[15] A. Ratto, M. Rigoli and L. Veron, 'Scalar curvature and conformal deformations of noncompact Riemannian manifolds', Math. Z. 225 (1997), 395-426.

School of Mathematics,

Statistics and Computer Science

University of New England

Armidale, NSW 2351

Australia

e-mail: ydu@turing.une.edu.au

Department of Mathematics

Qufu Normal University

Qufu, Shandong

Peoples Republic of China

e-mail: Ils@mail.qgfnu.edu.cn
Department of Mathematics

Qufu Normal University

Qufu, Shandong

Peoples Republic of China 